\title{
Somatic Embryogenesis and Plant Development in Centella asiatica L., a Highly Prized Medicinal Plant of the Tropics
}

\author{
Nirmal Joshee, Bipul K. Biswas, and Anand K. Yadav ${ }^{1}$ \\ Agricultural Research Station, Fort Valley State University, 1005 State \\ University Drive, Fort Valley, GA 31030
}

Additional index words. Centella asiatica, somatic embryogenesis, synseeds

\begin{abstract}
Past research experience with Centella asiatica micropropagation suggests a very high rate of contamination during the culture establishment stage. We demonstrate protocols for successful sterilization of Centella explants prepared from field-grown plants with an abundance of fungal and bacterial contamination. Sequential steps during sterilization and explant preparation process included a dip for $30 \mathrm{~s}$ in $70 \%$ ethyl alcohol, weak bleach treatment for $12 \mathrm{~min}$, and a $60-\mathrm{min}$ soak in plant preservative mixture before establishing cultures. We also report a reproducible system for somatic embryogenesis in Centella using leaf and stolon tip explants collected from naturally growing populations. Somatic embryos were induced within 3 to 4 weeks of culture in the dark on Murashige and Skoog (MS) medium supplemented with 2,4-dichlorophenoxyacetic acid (2,4-D). Initial embryogenic mass appeared as nodular callus, which eventually developed into actual somatic embryos exhibiting globular, heart-shaped, and cotyledonary stages. Leaves produced embryogenic calli at 2.26 and $4.52 \mu \mathrm{M}$ 2,4-D, whereas stolon tips were responsive only in the $9.04 \mu \mathrm{M}$ 2,4-D treatment. Withdrawal of 2,4-D/growth regulators from the induction medium resulted in the maturation and further development of the embryos into plantlets. Regular subculturing of the embryogenic calli into MS medium sustained their regenarability for more than 1 year. Somatic embryos were individually encapsulated in sodium alginate and calcium chloride-based encapsulation matrix to produce artificial or synthetic seeds (synseeds). Synseeds with $2 \%$ sodium alginate were found best for the survival and germination recorded after their storage at 5 to $8^{\circ} \mathrm{C}$ for 30 and 60 days. We report protocols for $C$. asiatica to reduce explant contamination before establishment of cultures on somatic embryo induction medium and efficient somatic embryogenesis to facilitate conservation and mass production of elite germplasm. This may further assist rapid dissemination of superior clones needed for research and commercial production.
\end{abstract}

Plant-based remedies have always been an integral part of traditional medicine throughout the world. Centella asiatica L. or Hydrocotyle asiatica (Apiaceae), commonly known as "Indian pennywort" or gotu kola, is a prostrate, faintly aromatic, and stoloniferous perennial tropical herb. In Ayurved, the old traditional medical system of India, it is reputed as a nervine tonic along with antibacterial, antifeedant, antileprotic, and wound-healing properties (Kakkar, 1988). The bioactive triterpenes of $C$. asiatica include a pair of saponins, asiatic acid and madecassic acid, and a pair of trisaccharides, asiaticoside and madecassoside (Schaneberg et al., 2003; Solet et al., 1998). Marquart et al. (1990) reported a positive correlation between a mixture of three terpenoids from C. asiatica and the collagen synthesis in human foreskin fibroblasts. In a recent study to identify the antiproliferative constituents

Received for publication 10 Nov. 2005. Accepted for publication $26 \mathrm{Feb} .2007$.

${ }^{1}$ To whom reprint requests should be addressed; e-mail yadava@fvsu.edu in C. asiatica, the methanol extracts of aerial parts inhibited the growth of human gastric adenocarcinoma, human uterine carcinoma, and murine melanoma cells in vitro (Yoshida et al., 2005).

In vitro culture techniques offer a viable tool for mass propagation of plants and conservation of rare, threatened, and endangered germplasm (Rao, 2004). In vitro culture studies on Centella have reported high incidences of microbial contamination, which drastically reduced explant survival (Tiwari et al., 2000). Micropropagation of $C$. asiatica through leaf and nodal explants has been reported earlier (Banerjee et al., 1999; Tiwari et al., 2000), and there is one report of somatic embryogenesis (Paramageetham et al., 2004). Plant regeneration through shoot morphogenesis is considered to have a multicellular origin that may result in the formation of genetically variable, chimeric plants. In contrast, somatic embryos are considered to arise either directly or indirectly from single cells and can give rise largely to genetically stable nonchimeric plants (Aly et al., 2002; Vasil and Vasil, 1994). Auxins (2,4-dichlorophenoxyacetic acid, indole ace- tic acid), have been perceived as the trigger molecules for the process of somatic embryo induction. Other reports indicate that these auxins need another auxin or cytokinin in combination for somatic embryo induction (Yong-Wook, 2000). Substitution for auxincytokinin requirement in the induction of somatic embryogenesis by thidiazuron (N-phenyl' N' -1，2，3-thidiazol-5-yl urea; Dropp, Bayer Crop Science, Research Triangle Park, N.C.) has been reported in geranium hypocotyl cultures (Visser et al., 1992), sugar beet (Zhang et al., 2001b), and Paulownia elongata (Ipekci and Gozukirmizi, 2003). Thidiazuron (TDZ) is reported to possess a strong cytokinin-like activity in a number of crops (Huetteman and Preece, 1993; Mok et al., 1987; Visser et al., 1992) and thus has been used in plant tissue culture studies. Somatic embryo induction in response to cytokinin alone is rare and less frequently reported as was shown in an elite variety of cotton and sugar beet (Zhang et al., 2001a). In addition to these plant growth regulators, other additives (glutamine, proline, casein hydrolysate, and a higher amount of sucrose) have positively influenced somatic embryogenesis. Synseeds have been used in the commercial propagation and conservation of plant germplasm (Gray and Purohit, 1991). They have been proposed and used as a system to preserve somatic embryos and other propagules for long- and short-term germplasm storage (Fang et al., 2004; Gangopadhyay et al., 2005; Mikula et al., 2005). This study reports a reproducible and rapid method for in vitro multiplication of $C$. asiatica through high-frequency somatic embryo proliferation from stolon tips as well as leaf explants followed by development of successful and viable synthetic seeds. We also provide information on the encapsulation of somatic embryos for conservation and low-temperature storability of germplasm.

\section{Materials and Methods}

Plant material and cultures. A patch measuring $2.5 \times 20 \mathrm{ft}$ of $C$. asiatica is growing in the specialty plant house (Fig. 1g) at Fort Valley State University. Actively growing stolons with nodes and leaves were collected during morning hours (08:00 to 09:00 HR). Older leaves and roots were trimmed off before disinfecting explants. Plant material was stirred for 20 min in tap water with a drop of Tween-20 and then placed under running tap water for $2 \mathrm{~h}$. These plants were passed through two changes of deionized water and then dipped in $70 \%$ ethyl alcohol for $1 \mathrm{~min}$ followed by one rinse in deionized water. The next step in sterilization was treatment of plant material in $15 \%$ commercial bleach (brand name Clorox, Oakland, Calif., sodium hypochlorite, available chlorine $6 \%$ with a final concentration of $0.9 \%$ ) for $12 \mathrm{~min}$ with constant stirring. Clorox-treated plant material was washed three times with autoclaved deionized water in a laminar flow hood and trimmed to remove bleached tissue and undesirable plant 

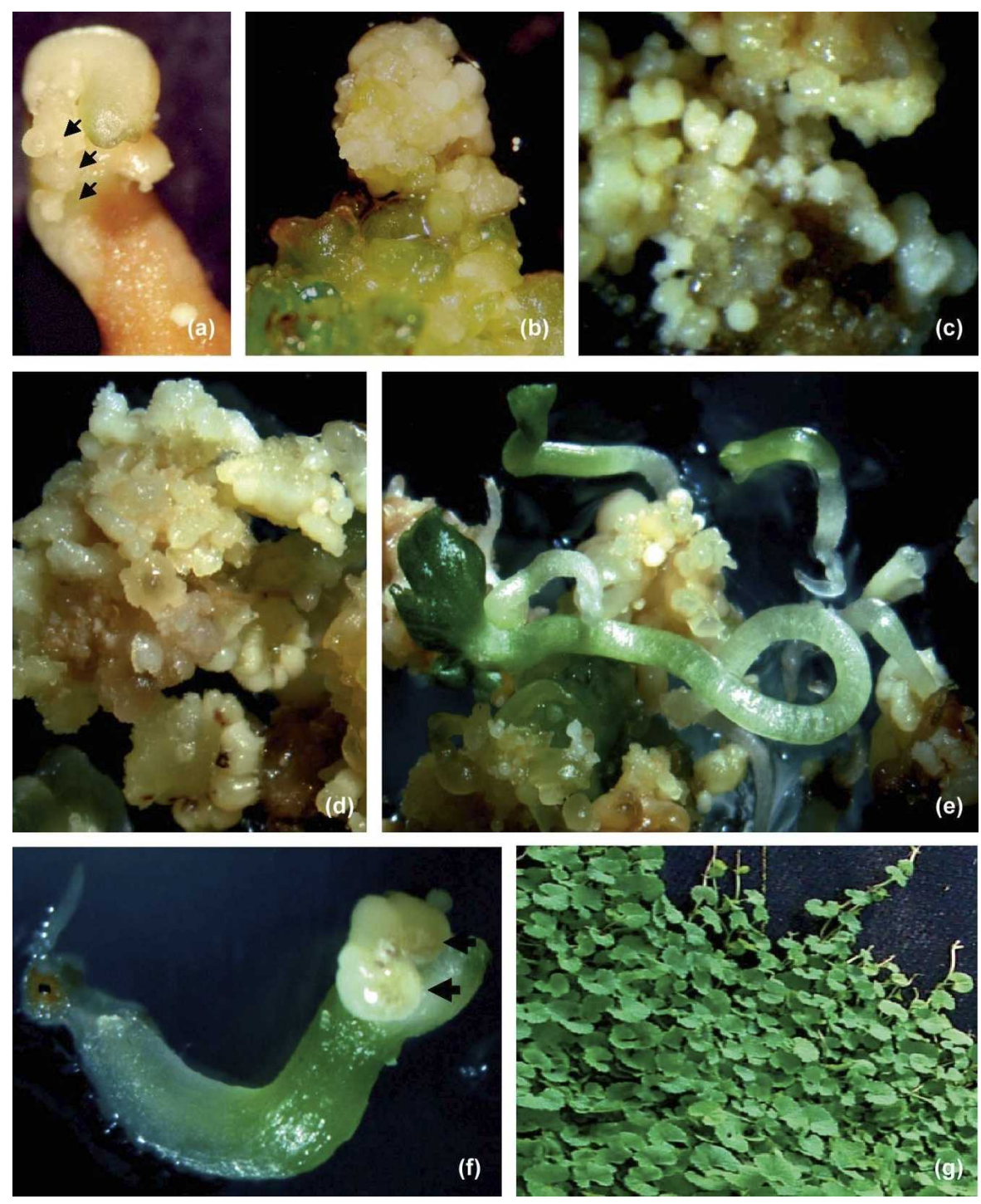

Fig. 1. Somatic embryo induction and secondary embryogenesis in Centella asiatica: (a) somatic embryo induction in stolon tip explant; arrows indicate somatic embryo formation. (b) Induction of nodular callus in young leaf explant. (c and d) conversion of nodular callus generated at the cut end of leaf into somatic embryos. (e) Asynchronous germination of somatic embryos exhibiting several developmental stages once cultures are transferred to growth regulator-free medium under light. Somatic embryos are seen developing cotyledons, leaf, shoot, and root. (f) A green developing embryo with secondary embryos. Secondary embryos are indicated with arrows. (g) Patch of Centella growing in a specialty plant house.

parts. Sterilized explants were submerged in 2\% PPM solution (Plant Cell Technology, Washington, D.C.) for 60 min before culturing them on somatic embryo induction medium (SEIM). To identify the most suitable explant for somatic embryo induction, 30 explants each of leaf (after trimming the margins, one leaf was cut into four to five pieces), internode (1.0 to $1.5 \mathrm{~cm}$ long), nodal segments, and stolon tips $(\approx 1.0 \mathrm{~cm}$ long) per treatment were inoculated on the Murashige and Skoog (MS) (1962) medium containing $3 \%$ sucrose, $0.25 \%$ Phytagel (Sigma, St. Louis) and various concentrations of plant growth regulators. All media were supplemented with $2 \mathrm{~mL} \cdot \mathrm{L}^{-1} \mathrm{PPM}$ after autoclaving to kill any contamination that might have escaped. Only unfurled leaves were selected to make explants. Each petri dish $(15 \times 90$ $\mathrm{mm}$ ) containing $25 \mathrm{~mL}$ medium was inoculated with four to five explants. All the cultures initiated for somatic embryogenesis were incubated in the dark at $25^{\circ} \mathrm{C} \pm 2{ }^{\circ} \mathrm{C}$.

Media composition and induction of somatic embryos. The SEIM used in this study was based on MS (1962) medium. The SEIM was supplemented with several concentrations of 2,4-dichlorophenoxyacetic acid (2,4-D), indole acetic acid (IAA) and TDZ to investigate the role of auxins and TDZ in somatic embryo induction. Four concentrations of 2,4-D $(0.45,2.26,4.52$, and $9.04 \mu \mathrm{M})$, three concentrations of IAA $(0.57,2.85$, and $5.71 \mu \mathrm{M})$, and four concentrations of TDZ $(0.22,0.45,2.27$, and 4.54 $\mu \mathrm{M})$ were tested individually for somatic embryo induction (Table 1). In addition to these individual concentrations, three treat-
Table 1. Somatic embryo induction in Centella asiatica in response to various growth regulators added to culture media individually and in combination

\begin{tabular}{llc}
\hline Treatments $(\mu \mathrm{M})$ & $\begin{array}{c}\text { Responsive } \\
\text { explant }\end{array}$ & $\begin{array}{c}\text { Somatic embryo } \\
\text { induction }\end{array}$ \\
\hline Control & & \\
2,4-D 0.45 & & \\
2,4-D 2.26 & Leaf & + \\
2,4-D 4.52 & Leaf & ++++ \\
2,4-D 9.04 & Stolon tip & ++
\end{tabular}

IAA 0.57

IAA 2.85

IAA 5.71

2,4-D $0.45+$

IAA 0.57

2,4-D $2.26+$

IAA 2.85

2,4-D $4.52+$

IAA 5.71

TDZ 0.22

TDZ 0.45

TDZ 2.27

TDZ 4.54

2,4-D = 2,4-dichlorophenoxyacetic acid; IAA = indole acetic acid; TDZ $=$ thidiazuron.

ments of 2,4-D and IAA in combination were also investigated $(0.45+0.57,2.26+22.85$, and $4.52+5.71 \mu \mathrm{M})$. All these treatments were also supplemented with $200 \mathrm{mg} / \mathrm{L}$ glutamine and $100 \mathrm{mg} / \mathrm{L}$ casein hydrolysate before autoclaving. The $\mathrm{pH}$ of the medium was adjusted to 5.7 to 5.8 before autoclaving. As a gelling agent, $2.5 \mathrm{~g} \cdot \mathrm{L}^{-1}$ Phytagel (Sigma) was added to the medium and then sterilization of the culture medium was carried out by autoclaving at $121{ }^{\circ} \mathrm{C}$ for $20 \mathrm{~min}$. All plant growth regulators were filter-sterilized and added to the medium after autoclaving.

Cultures were maintained at $25^{\circ} \mathrm{C} \pm 2{ }^{\circ} \mathrm{C}$ in the dark for 3 to 4 weeks until nodular callus with early stages of somatic embryos was seen. Somatic embryo-producing embryogenic masses were isolated from responsive explants after 1 month of proliferation and transferred to MS medium supplemented with $0.45 \mu \mathrm{M} 2,4-\mathrm{D}$ in the dark to harvest more somatic embryos for encapsulation experiments. Furthermore, somatic embryos for germination and survival studies were moved to growth regulator-free MS medium under 14-h photoperiod light with a photon flux of $\approx 60 \mu \mathrm{mol} \cdot \mathrm{m}^{-2} \cdot \mathrm{s}^{-1}$ provided by cool-white fluorescent tubes.

Development of somatic embryos. Once the induction of embryogenic callus and somatic embryos was evident, the calli were transferred to MS medium supplemented with $0.45 \mu \mathrm{M}$ 2,4-D. Calli in this medium kept proliferating making globular and heartshaped somatic embryos (SEs). These calli were subcultured every 3 to 4 weeks to the same medium for the production of SEs and were kept in the dark. For further development and their germination, SEs were subcultured into hormone-free MS medium containing 3\% sucrose. Somatic embryos were cultured for 4 weeks under a 14-h photoperiod at $25{ }^{\circ} \mathrm{C} \pm 2{ }^{\circ} \mathrm{C}$ and observed periodically to record various stages in the development. Photomicrography was 
done using an Olympus SZX12 microscope (Olympus America, Center Valley, Pa.).

Synthetic seed formation. For encapsulation purposes, we tested $1 \%, 2 \%$, and $3 \%$ sodium alginate $(\mathrm{w} / \mathrm{v})$ dissolved in basal MS medium containing 3\% sucrose. Somatic embryos (1.0 to $2.0 \mathrm{~mm}$ long, mainly heartshaped and bipolar stage) subcultured to hormone-free MS medium were used for encapsulation. Individual somatic embryos were pulled into a pipette with $0.4-\mathrm{mm}$ i.d. containing 0.15 to $0.20 \mathrm{~mL}$ alginate mixture. Alginate-coated somatic embryos were then dropped into 50 and $80 \mathrm{~mm}(\mathrm{w} / \mathrm{v})$ calcium chloride solution. Each drop containing a single embryo was maintained in calcium chloride for 10,30 , or $60 \mathrm{~min}$. After the incubation periods, the beads were recovered by decanting the calcium chloride solution and washing three times with MS basal solution. For each treatment, at least 50 somatic embryos were encapsulated. Nonencapsulated embryos were taken as controls. The survival and germination of both encapsulated and control embryos were recorded after 6 weeks.

Low-temperature storage and synseed germination. Experiments were carried out to evaluate the effect of cold storage on the germination capacity of encapsulated SEs. The encapsulated embryos were transferred onto petri dishes $(15 \times 90 \mathrm{~mm})$ containing $25 \mathrm{~mL}$ MS basal medium and were sealed. These sealed petri dishes with 10 synseeds in each were left for 30 and $60 \mathrm{~d}$ in the dark at 5 to $8{ }^{\circ} \mathrm{C}$ for storage. For control, embryos without encapsulation were maintained at room temperature in the dark and were placed in light after 30 and $60 \mathrm{~d}$ along with coldtreated synseeds.

\section{Results}

Plant materials and cultures. Our results indicate that cleaning plants under running water, a dip in $70 \%$ ethanol, and then a dilute commercial bleach treatment helped the decontamination process. Furthermore, a 60 -min soak in a 2\% PPM solution before culture establishment and all culture media supplemented with $2 \mathrm{~mL} \cdot \mathrm{L}^{-1} \mathrm{PPM}$ helped to control fungal and bacterial contaminants. This procedure resulted in the survival and establishment of more than $90 \%$ of explants inoculated on the SEIM.

Media composition and induction of somatic embryos. In our pilot experiments, MS medium with additives (glutamine and casein hydrolysate, data not shown) exhibited better survival and response of explants. Thus, all experiments were carried out by supplementing MS medium with $200 \mathrm{mg} \cdot \mathrm{L}^{-1}$ glutamine and $100 \mathrm{mg} \cdot \mathrm{L}^{-1}$ casein hydrolysate. The results obtained (Table 1) show that somatic embryos were induced on both stolon tips and leaf explants when cultured on MS basal medium supplemented with 2,4-D and 2,4-D + IAA. Onset of nodular callus and development of SEs was observed on explants after 3 to 4 weeks of culturing in the dark. Younger unfurled leaves showed better response than the older open ones. Leaves produced embryogenic calli at 2.26 and $4.52 \mu \mathrm{M}$ concentration of $2,4-\mathrm{D}$, whereas stolon tips were responsive only at $9.04 \mu \mathrm{M}$ 2,4-D treatment (Table 1). In leaves, $4.52 \mu \mathrm{M}$ 2,4-D induced more prolific response than lower concentrations of 2,4-D used in this study and later only this concentration was used to induce embryogenic calli. Leaf explants cultured on SEIM turned brown after 3 to 4 weeks and cream-colored embryogenic mass of callus was initiated at the cut end (Fig. 1b, c). The tissue swelled, developed typical embryogenic nodular callus, and formed many tiny somatic embryos at different stages of development at the cut ends of leaf explants. Proliferation of nodular embryogenic callus is evident in the leaf explant, and this nodular mass was transferred to $0.45 \mu \mathrm{M}$ 2,4-D containing media for further proliferation (Fig. 1b). Very few stolon tips that responded (less than 5\%) also developed somatic embryos on the surface of the explant without callus induction (Fig. 1a). The embryogenic tissues developed globular structures and then progressed to
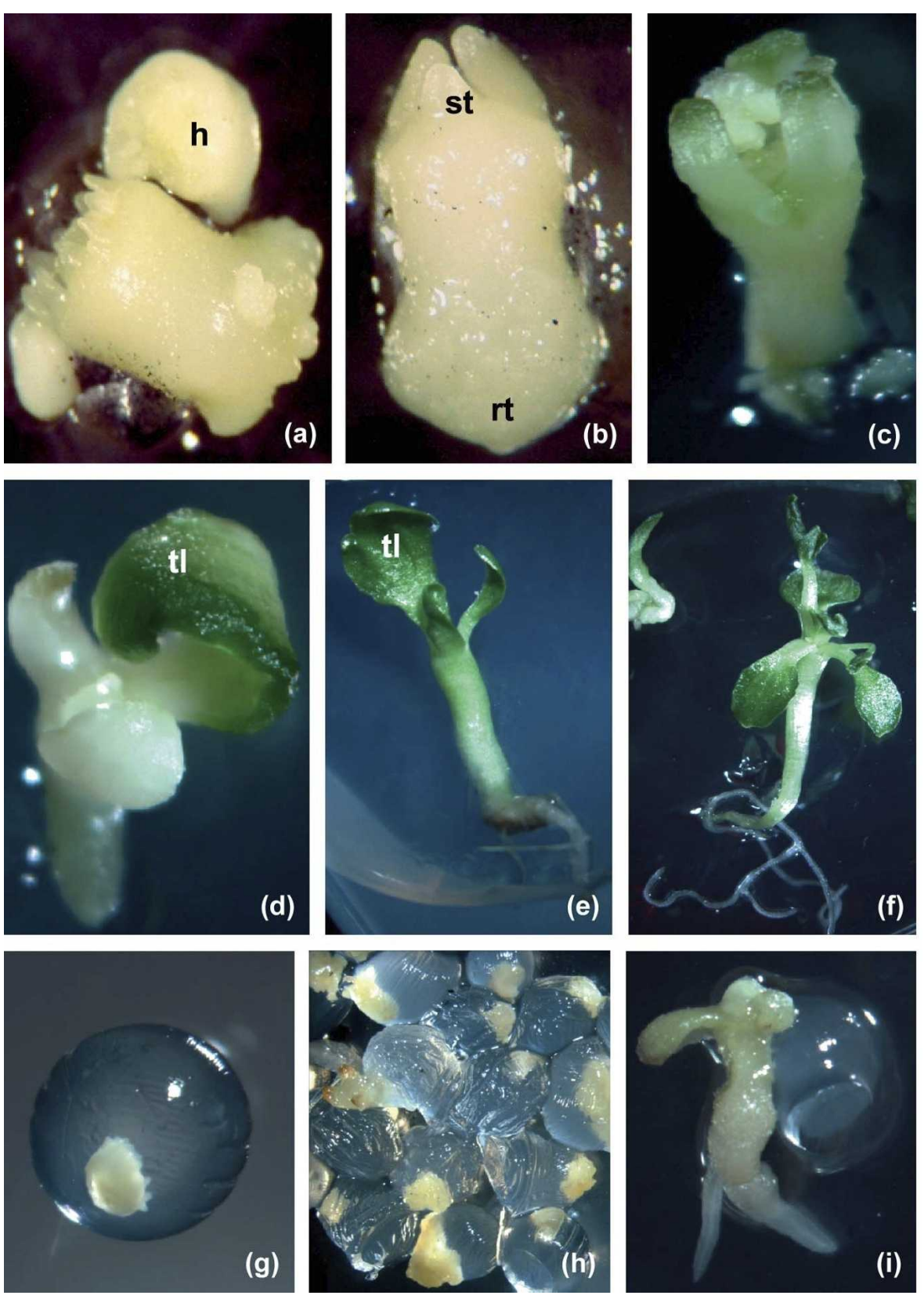

Fig. 2. Somatic embryo development and encapsulation studies in Centella asiatica: (a) Cluster of somatic embryos (SEs) in different developmental stages with a heart-shaped (h) structure and elongating SE developing polarity. (b) Bipolar embryo with cotyledons, shoot tip (st), and root tip (rt). (c) Greening of bipolar embryo when transferred to a 14-h photoperiod condition. (d) Unfurling of first true leaf. (e) Greening of true leaf (tl) and cotyledons. Root elongation is also evident at this stage. (f) More true leaves develop and internode elongation takes place. (g) Single heart-shaped embryo encapsulated in $2 \%$ sodium alginate. (h) A batch of somatic embryos stored at 5 to $8{ }^{\circ} \mathrm{C}$ for $60 \mathrm{~d}$. (i) Germinating encapsulated somatic embryo in basal Murashige and Skoog medium after $60 \mathrm{~d}$ of storage at 5 to $8^{\circ} \mathrm{C}$. 
heart- and torpedo-shaped stages within 10 to $14 \mathrm{~d}$. Periodic subculturing of embryogenic masses to PGR-free medium increased the frequency of somatic embryo formation (Fig. 1d). Repetitive somatic embryogenesis was frequent and led to the formation of many small embryoids. Secondary embryogenesis was also observed on developing embryos (Fig. 1f).

Somatic embryos differentiated into plantlets with cotyledons, hypocotyls, and radical when placed on plant growth regulator-free MS basal medium and cultured under a 14-h photoperiod (Fig. 1e). These somatic embryos follow an asynchronous mode of development because different developmental stages could be observed in the same culture mass (Fig. 1e). A fully developed normal embryo is presented in Figure 1f. There was no somatic embryo induction in the treatments in which medium was supplemented with IAA and TDZ as the only PGR source (Table 1). In a few unfurled leaf explants, $2.26 \mu \mathrm{M} 2,4-\mathrm{D}$ and $2.85 \mu \mathrm{M}$ IAA in combination produced somatic embryos, whereas other combination treatments failed to trigger any embryogenic response (Table 1).

Somatic embryo development, germination, and plantlet formation. Somatic embryos at this point could be germinated on a PGRfree MS basal medium progressing through globular-, heart-, and torpedo-shaped developmental stages or would be directed to produce more somatic embryos in MS medium supplemented with $0.45 \mu \mathrm{M}$ 2,4-D. Various stages, characteristic of dicot embryogeny, can be clearly seen in a culture of developing embryos (Fig. 2a-f). On a growth regulator-free medium, under a 14-h long photoperiod, cream-colored somatic embryos developed shoot and root apices in 3 to 4 weeks, initiated elongation of cotyledons, and turned green (Fig. 2a-d). It took another 2 to 3 weeks for unfurling first true leaves, elongation of hypocotyls, and induction of roots (Fig. 2e). In Figure 2f, extension of epicotyl and shoot is evident along with additional root growth.

Synthetic seed germination. The concentration of sodium alginate $(1 \%, 2 \%$, and $3 \%)$ and $\mathrm{CaCl}_{2} \cdot 2 \mathrm{H}_{2} \mathrm{O}$ (calcium chloride) solutions (50 and $80 \mathrm{~mm}$ ) and the exposure of alginate solution containing somatic embryo to calcium chloride solution for complexation (10, 30 , and $60 \mathrm{~min}$ ) needed to make firm bead were studied. Somatic embryos encapsulated with $2 \%$ sodium alginate prepared in MS salt solution and submerged 30 to $60 \mathrm{~min}$ in $80 \mathrm{~mm}$ calcium chloride for hardening produced seeds with a coat firm enough for handling and allowing synseeds to develop into plantlets (Fig. 2g). We found that 30-min exposure in $80 \mathrm{~mm}$ calcium chloride solution was enough to produce firm synseeds for studying the effect of cold storage on survival and germination.

Low-temperature storage and germination of synseeds. Synseeds using different concentrations of sodium alginate were prepared and kept at 5 to $8^{\circ} \mathrm{C}$ (Fig. 2h) in a petri dish containing MS basal medium for 30 and $60 \mathrm{~d}$. After this storage period, synseeds were taken out and placed on MS basal medium in petri dishes. These plates were kept under a 14-h photoperiod at $25{ }^{\circ} \mathrm{C} \pm 2{ }^{\circ} \mathrm{C}$ and germination of embryo was recorded. A $2 \%$ sodium alginate concentration used to make synseeds resulted in higher germination than at $1 \%$ and $3 \%$ concentrations in this investigation (Table 2). Germinating synseeds were allowed to grow and develop strong root and shoot systems (Fig. 2f). At this stage, plants were gently removed from culture vessel and transferred to a mist chamber for hardening in the greenhouse. After $7 \mathrm{~d}$, pots containing plants were removed from the mist chamber and placed on a greenhouse bench for further growth. Of 10 plants acclimatized, six survived and developed into normal plants.

Abnormal embryos. Different stages of embryogenic structures, normal and abnormal, were observed to occur on the same medium. Both higher concentrations of 2,4-D and prolonged incubation in induction medium induced abnormal somatic embryos. Occurrence of various abnormal structures such as leaf-like structures and increased number of cotyledons were observed. Abnormal embryos exhibited a poor rate of regeneration into a complete plantlet.

\section{Discussion}

Because the Centella plant has a creeping habit, decontaminating the explants to establish initial cultures is challenging. We present a protocol that is simple and works without using a hazardous chemical mercuric chloride and antibiotics as has been used before (Tiwari et al., 2000). Careful analysis of cultures during the induction phase revealed that both the direct and indirect modes of somatic embryo development were present. Using the same medium and plant growth regulators, leaf and stolon explants differed in somatic embryo induction because on stolon, somatic embryos originated directly, whereas on leaves, it was through a brief intervening callus phase. This situation has been previously reported (Gray and Conger, 1985; Grieb et al., 1997). It seems that the direct embryogenesis generally proceeds from cells already determined for embryogenic development before explantation (Fig. 1a). The so-called preembryogenic determined cells only required growth regulators or favorable conditions to allow their entry into cell division and expression of embryogenesis. By contrast, indirect embryogenesis nominally required redetermination of differentiated cells, callus proliferation, and the acquisition or development of the embryogenically determined state.

The present study establishes that 2,4-D is one of the most important auxins involved in the somatic embryogenesis process. Somatic embryo induction in the combination treatment may possibly be attributed to the presence of 2,4-D alone because individual IAA treatments did not register any response (Table 1). In a recent study on $C$. asiatica, somatic embryos were formed on a medium supplemented with $9.29 \mu \mathrm{M}$ kinetin in combination with $2.26 \mu \mathrm{M} 2,4-\mathrm{D}$ suggesting the central role 2,4-D plays in this process. In addition to this, physiological status of explant is also very important because fully opened mature leaves responded poorly in comparison with young unfurled leaves.

Application of TDZ has been reported to induce an array of cultural responses ranging from callus induction to formation of somatic embryos in many plants (Huetteman and Preece, 1993; Ipekci and Gozukirmizi, 2003; Mok et al., 1987; Murthy et al., 1998; Visser et al., 1992; Zhang et al., 2001b). Somatic embryogenesis induced under the influence of TDZ on the lower surfaces of cotton cotyledons were less genotype-dependent than when BA was used (Zhang et al., 2001a). In Paulownia elongata, the highest induction frequencies of somatic embryos were obtained on MS medium supplemented with 3\% sucrose, $0.6 \%$ Phytagel, $500 \mathrm{mg} \cdot \mathrm{L}^{-1}$ casein hydrolysate, and $10 \mathrm{mg} \cdot \mathrm{L}^{-1} \mathrm{TDZ}$. Subsequent withdrawal of TDZ from the induction medium resulted in the maturation and growth of the embryos into plantlets on MS basal media (Ipekci and Gozukirmizi, 2003). Glutamine and casein hydrolysate have been linked to assisting somatic embryogenesis in some of the plants (Dai et al., 2004; Gray and Conger, 1985).

Table 2. Germination of somatic embryos of Centella asiatica in response to different concentrations of sodium alginate and low temperature storage duration before being transferred to hormone-free MS medium.

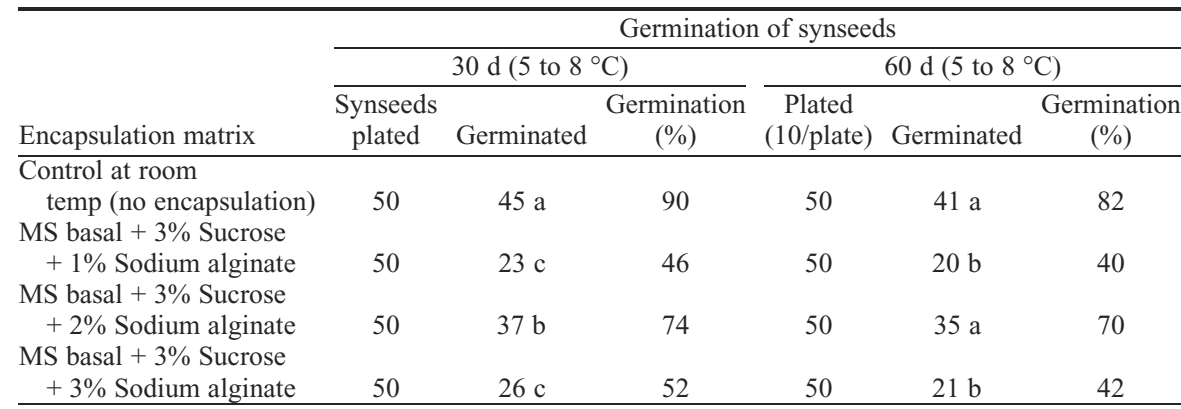

Data were analyzed by using MIXED model of SAS. Means were separated by least square means test $(P \leq 0.05)$.

MS $=$ Murashige and Skoog. 
The germination percentage of encapsulated somatic embryos is affected significantly by the concentration of sodium alginate, and it has been reported in other plants too (Castillo et al., 1998; Lakshmana Rao and Singh, 1991; Malabadi and van Staden, 2005). In the present study, synseeds with $2 \%$ sodium alginate coat did the best in terms of germination in comparison with controls after 30 and $60 \mathrm{~d}$ of cold treatment (Table 2). This study indicates that lowtemperature storage could be an important conservation tool. Our preliminary result of $60 \%$ survival of plants raised from synseeds indicates the possibility of it as a good conservation tool. Further study using cryoprotectants and different types of soluble and insoluble sugars in the encapsulation matrix may be helpful for longer storage and better germination and survival. In Paulownia elongata, a 3\% sodium alginate concentration provided uniform encapsulation of the embryos with survival and germination frequencies of $73.7 \%$ and $53.3 \%$, respectively. Storage at $4{ }^{\circ} \mathrm{C}$ for 30 or $60 \mathrm{~d}$ significantly reduced the survival and complete germination frequencies of both encapsulated and nonencapsulated embryos relative to those of nonstored somatic embryos (Ipekci and Gozukirmizi, 2003). In a recent study on Pinus patula, synseeds could be stored at $2{ }^{\circ} \mathrm{C}$ for $120 \mathrm{~d}$ without a reduction in germination (Malabadi and van Staden, 2005). In Solanum melongena, $3 \%$ sodium alginate and $75 \mathrm{~mm}$ calcium chloride was found to be optimal for encapsulation studies (Lakshmana Rao and Singh, 1991).

In vitro propagated plants of many important medicinal species were found to be uniform, showing less variation in their content of secondary metabolites than their wild/cultivated counterparts (Yamada et al., 1991). Micropropagated plants can be used to supplement the natural stock of plants in wild populations as well as to provide a ready supply to the herbal medicinal trade, thereby alleviating pressure on existing resources already overexploited by uncontrolled harvesting.

\section{Literature Cited}

Aly, M.A.M., B. Rathinasabapathi, and K. Kelly. 2002. Somatic embryogenesis in perennial statice Limonium bellidifolium Plumbaginaceae. Plant Cell Tiss. Org. Cult. 68:127-135.

Banerjee, S., M. Zehra, and S. Kumar. 1999. In vitro multiplication of Centella asiatica, a medicinal herb from leaf explants. Curr. Sci. 76:147-148

Castillo, B., M.A.L. Smith, and U.L. Yadav. 1998. Plant regeneration from encapsulated somatic embryos of Carica papaya. Plant Cell Rep. 17:172-176.

Dai, J., A.V. Wagner, and S.A. Merkle. 2004. Enhancing the production of hybrid yellowpoplar and hybrid sweetgum embryogenic cultures. In Vitro Cell. Dev. Biol. Plant 40:376383.

Fang, J.-Y., A. Wetten, and P. Hadley. 2004. Cryopreservation of cocoa (Theobroma cacao L.) somatic embryos for long-term germplasm storage. Plant Sci. 166:669-675.

Gangopadhyay, G., T. Bandyopadhyay, R. Poddar, S.B. Gangopadhyay, and K.K. Mukherjee. 2005. Encapsulation of pineapple micro shoots in alginate beads for temporary storage. Curr. Sci. 88:972-977.

Gray, D.J. and B.V. Conger. 1985. Influence of dicamba and casein hydrolysate on somatic embryo number and culture quality in cell suspensions of Dactylis glomerata (Gramineae). Plant Cell Tiss Org. Cult. 4:123-133.

Gray, D.J. and A. Purohit. 1991. Somatic embryogenesis and development of synthetic seed technology. Crit. Rev. Plant Sci. 10:33-61.

Grieb, B., F. Schafer, K. Nezamabadi, B. ArnholtSchmitt, and K.H. Neumann. 1997. Changes in soluble proteins and phytohormone concentrations of cultured carrot petiole explants during induction of somatic embryogenesis (Daucus carota, L.). Angewandte Botanik 71:94-103.

Huetteman, C.A. and J.E. Preece. 1993. Thidiazuron: A potent cytokinin for woody plant tissue culture. Plant Cell Tiss. Org. Cult. 33: $105-119$.

Ipekci, Z. and N. Gozukirmizi. 2003. Direct somatic embryogenesis and synthetic seed production from Paulownia elongata. Plant Cell Rep. 22:16-24.

Kakkar, K.K. 1988. Mandukaparni-medicinal uses and therapeutic efficacy. Indian Drugs 26:92-97.

Lakshmana Rao, P.V. and B. Singh. 1991. Plantlet regeneration from encapsulated somatic embryos of hybrid Solanum melongena L. Plant Cell Rep. 10:7-11.

Malabadi, R.B. and J. van Staden. 2005. Storability and germination of sodium alginate encapsulated somatic embryos derived from the vegetative shoot apices of mature Pinus patula trees. Plant Cell Rep. 82:259-265.

Marquart, F.X., G. Bellon, P. Gillery, Y. Wegrowski, and J.P. Borel. 1990. Stimulation of collagen synthesis in fibroblast cultures by a triterpene extracted from Centella asiatica. Conn Tissue Res 24:107-120.

Mikula, A., A. Fiuk, and J.J. Rybczynski. 2005. Induction, maintenance, and preservation of embryogenic competence of Gentiana cruciata L. cultures. Acta Biologica Cracoviencia 47: 227-236.

Mok, M.C., D.W.S. Mok, J.E. Turner, and C.V. Mujer. 1987. Biological and biochemical effects of cytokinin-active phenylurea derivatives in tissue culture systems. HortScience 22:1194-1197.

Murashige, T. and F. Skoog. 1962. A revised medium for rapid growth and bioassays with tobacco tissue cultures. Physiol. Plant. 15:473497.

Murthy, B.N.S., S.J. Murch, and P.K. Saxena. 1998. Thidiazuron: A potential regulator of in vitro plant morphogenesis. In Vitro Cell. Dev. Biol. Plant 34:267-275.

Paramageetham, Ch., G.P. Babu, and J.V.S. Rao. 2004. Somatic embryogenesis in Centella asiatica L. an important medicinal and neutraceutical plant of India. Plant Cell Tiss. Org. Cult. 79:19-24.

Rao, N.K. 2004. Plant genetic resources: Advancing conservation and use through biotechnology. Afr. J. Biotechnol. 3:136-145.

Schaneberg, B.T., J.R. Mikell, E. Bedir, and I.A. Khan. 2003. An improved HPLC method for quantitative determination of six triterpenes in Centella asiatica extracts and commercial products. Pharmazie 58:381-384.

Solet, J.M., A. Simon-Ramiasa, L. Cosson, and J.L. Guignard. 1998. Centella asiatica (L.) Urban. (Pennywort): Cell culture, production of terpenoids and biotransformation capacity. In: Y.P.S. Bajaj (ed.). Biotechnology in agriculture and forestry, Vol. 41. Medicinal and Aromatic Plants X Springer-Verlag, Berlin.

Tiwari, K.N., N.C. Sharma, V. Tiwari, and B.D. Singh. 2000. Micropropagation of Centella asiatica (L.), a valuable medicinal herb. Plant Cell Tissue Organ Cult. 63:179-185.

Vasil, I.K. and V. Vasil. 1994. In vitro culture of cereal and grasses, p. 293-312. In: I.K. Vasil and T.A. Thorpe (eds.). Plant cell and tissue culture. Kluwer Academic Publishers, Dordrecht, The Netherlands.

Visser, C., J.A. Qureshi, R. Gill, and P.K. Saxena. 1992. Morphoregulatory role of thidiazuron. Plant Physiol. 99:1704-1707.

Yamada, Y., Y. Shoyama, I. Nishioka, H. Kohda, A. Namera, and T. Okamoto. 1991. Clonal micropropagation of Gentiana scabra Bunge var. buergeri Maxim. and examination of the homogeneity concerning the gentiopicroside content. Chem. Pharm. Bull. (Tokyo) 39:204 206.

Yong-Wook, K. 2000. Somatic embryogenesis in Quercus acutissima, p. 671-685. In: S.M. Jain, P.K. Gupta, and R.J. Newton (eds.) Somatic embryogenesis in woody plants. Kluwer Academic Publishers, Dordrecht, The Netherlands.

Yoshida, M., M. Fuchigami, T. Nagao, H. Okabe, K. Matsunaga, J. Takata, Y. Karube, R. Tsuchihashi, J. Kinjo, K. Mihashi, and T. Fujioka. 2005. Antiproliferative constituents from Umbellifereae plants VII. Active triterpenes and rosmarinic acid from Centella asiatica. Biol. Pharm. Bull. 28:173-175.

Zhang, B.-H., R. Feng, F. Liu, and Q. Wang. 2001a. High frequency somatic embryogenesis and plant regeneration of an elite Chinese cotton variety. Bot. Bull. Acad. Sin. 42:9-16.

Zhang, C.-L., D.-F. Chen, M.C. Elliott, and A. Slater. 2001b. Thidiazuron-induced organogenesis and somatic embryogenesis in sugar beet (Beta vulgaris L.). Vitro Cellular and Plant Biol. Plant. 37:305-310. 